\title{
In Celluloidgewittern
}

\section{Die mediale Konstruktion von Wissenschaft und Technik als Paradigma des Ersten Weltkrieges*}

\author{
von Silke Fengler und Stefan Krebs
}

\begin{abstract}
Überblick
Der Beitrag setzt sich kritisch mit der Inszenierung von Wissenschaft und Technik im Ersten Weltkrieg in aktuellen zeithistorischen TV-Dokumentationen auseinander. Deren problematische Kernaussage - natur- und ingenieurwissenschaftliche Forschung und Entwicklung hätten dem Ersten Weltkrieg eine völlig neue, moderne Dimension verliehen -, wird im ersten Teil kritisch beleuchtet. Dabei zeigt sich, dass diese tatsächlich nur einen Teilaspekt des vielschichtigen, normativ aufgeladenen Phänomens der Moderne darstellen, die Filmemacher also mit einem verengten Modernitätsbegriff arbeiten.

Der zweite Teil des Beitrags beschäftigt sich mit der Inszenierung des historischen Bildmaterials und fragt nach den Gründen für die von den heutigen TV-Autoren gewählte Darstellungsform. Die durchgehende Illustrierung mit zeitgenössischen Filmaufnahmen hat gravierende Folgen für die Erzählinhalte: eine ausführliche Bildanalyse zeigt, dass die Autoren weitgehend den in die Bilder eingravierten Diskursen folgen. Der Verzicht auf eine quellenkritische Analyse hat die oben erwähnte verkürzte Darstellung des Ersten Weltkrieges zur Folge. Die Rekombination zeitgenössischer Aufnahmen trägt auch dazu bei, eine - von heutigen ästhetischen Konzepten geprägte - Idee des modernen Krieges zu perpetuieren. Und schließlich knüpft die spezifische Erzählform über den Ersten Weltkrieg an aktuelle Strömungen der bundesdeutschen Erinnerungskultur an.
\end{abstract}

\begin{abstract}
This paper is a critical discussion of the way present day historical TV documentaries portray the science and technology of the First World War. Their problematic central statement, i.e. that research and development in natural science and engineering lent to the First World War a completely new, modern dimension is carefully examined in the first part. It turns out that these documentaries actually represent only one aspect of the multi-faceted, normatively charged phenomenon of modernity, and that the authors employ a limited notion of modernity.

* Wir danken unseren Kollegen Dipl.-Ing Alexander Faridi und Dr. Werner Tschacher für ihre Anregungen und die kritische Durchsicht unseres Manuskriptes.
\end{abstract}


The second part of the paper deals with the portrayal of historical pictorial material, inquiring into the reasons for the form of representation chosen by today's TV authors. The continuous use of historical illustrations has considerable consequences for the stories' content: a detailed image analysis shows that the authors allow themselves to be led by the discourses engraved in the pictures themselves. This renouncement of a critical analysis of source material entails exactly the aforementioned abridged view of World War One. The recombination of contemporary photographs - shaped by today's aesthetic concepts - perpetuates the idea of the modern war. Finally, the specific narration is connected to the memory culture of today's German Federal Republic.

Mit dem neunzigsten Jahrestag des Kriegsausbruchs rückte im Sommer letzten Jahres der Erste Weltkrieg kurzzeitig ins Zentrum des deutschen Medieninteresses. Auf ARTE und in der ARD liefen aus diesem Anlass mehrere zeithistorische Dokumentationen zu unterschiedlichen Aspekten dieses als modern apostrophierten Krieges. Die ARD zeigte eine fünfteilige Serie „Der

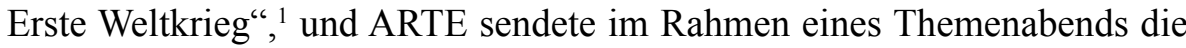
90minütige Dokumentation „Der Moderne Krieg“.2 Das Filmmaterial der ARTE-Produktion ist in großen Teilen dem zweiten und dritten Teil der ARDSerie - „Gashölle Ypern“ und „Alptraum Verdun“ - entnommen. In beiden Dokumentationen wird die Geschichte des Ersten Weltkrieges vorwiegend aus deutscher Perspektive geschildert. Dementsprechend entwickelt der vorliegende Beitrag seine Thesen vor dem Hintergrund der deutschen Kriegserfahrung.

Die zentrale These der TV-Beiträge lautet, dass erstmals eine enge Verflechtung zwischen natur- und ingenieurwissenschaftlicher Forschung und der Entwicklung neuer Waffensysteme bestand. Und die industrielle Massenfertigung von Rüstungsgütern und ihr ungehemmter Einsatz seien dafür verantwortlich gewesen, dass die Kriegsführung eine völlig neue, eben moderne, Dimension erreichte. ${ }^{3}$

1 Fünfteilige Fernsehproduktion Der Erste Weltkrieg, Deutschland 2004: Mythos Tannenberg, Buch und Regie von Susanne Stenner, Erstausstrahlung 26.7.2004, ARD; Gashölle Ypern, Buch und Regie von Heinrich Billstein, Erstausstrahlung 2.8.2004, ARD; Alptraum Verdun, Buch und Regie von Mathias Haentjes und Werner Biermann, Erstausstrahlung 4.8.2004, ARD; Schlachtfeld Heimat, Buch und Regie von Anne Roerkohl, Erstausstrahlung 9.8.2004, ARD; Trauma Versailles, Buch und Regie von Gabriele Trost, Erstausstrahlung 16.8.2004, ARD.

2 1914-1918: Der Moderne Krieg, Buch und Regie von Heinrich Billstein und Mathias Haentjes, Deutschland 2004, Erstausstrahlung 30.7.2004, ARTE.

3 Vgl. exemplarisch die Eingangssequenz: 1914-1918: Der Moderne Krieg, 00:01:5400:03:54. 
Warum greifen die Filmemacher gerade Wissenschaft und Technik als Inbegriff für die Modernität des Ersten Weltkrieges heraus? Der Aufsatz zeigt anhand einer ausführlichen Bildanalyse, dass die Autoren mit dieser Deutung weitgehend unreflektiert der inneren Logik zeitgenössischer Filmaufnahmen folgen. Weiter nutzen sie Bildauswahl und Rekombination der Aufnahmen, um ihre - von heutigen ästhetischen Konzepten geprägte - Idee des modernen Krieges zu veranschaulichen. Und schließlich binden sie den Ersten Weltkrieg an aktuelle Strömungen der bundesdeutschen Erinnerungskultur an. Bei der Auseinandersetzung mit diesen Fragen geht es letztlich darum, den Umgang mit Bildern in der Geschichtswissenschaft und ihre Verwendung zur medialen Konstruktion von Vergangenheit zu thematisieren.

Zunächst soll jedoch eingehender die Frage erörtert werden, inwieweit der Erste Weltkrieg überhaupt angemessen als modern bezeichnet werden kann. Dabei geht es nicht darum, die Auswirkung der sich rasant entwickelnden Waffentechnik und Rüstungsindustrie auf das Kriegsgeschehen oder die industrielle Dimension des Krieges in Abrede zu stellen. Vielmehr soll zweierlei deutlich werden: zum einen, dass Wissenschaft und Technik nur einen Teilaspekt des vielschichtigen Phänomens der Moderne darstellen. Zum anderen die Problematik, den Begriff der Modernisierung losgelöst von seiner positiv besetzten, normativen Konnotation zu verwenden. Erst vor dem Hintergrund der folgenden Überlegungen zeigt sich daher, dass die Filmemacher mit einem vielfach verengten Modernitätsbegriff arbeiten.

\section{Zur Modernität des Ersten Weltkrieges}

Wie die geschichtswissenschaftliche Kontroverse „Nationalsozialismus und Modernisierung“ gezeigt hat, sind die Begriffe modern, Modernität und Modernisierung problematisch, können sie doch mit ganz unterschiedlichen Bedeutungen aufgeladen sein. In der einfachsten Form wird das Adjektiv modern schlicht als Synonym für neu bzw. zum ersten Mal verwendet. Darüber hinaus erhält es keine weitere explizite Bedeutungszuschreibung. Die Begriffe Modernität und Modernisierung beziehen sich entweder auf komplexe gesellschaftliche Wandlungsprozesse oder lediglich auf isolierte Teilsysteme innerhalb einer Gesellschaft. Nach Norbert Frei sollte Modernisierung als normativer Begriff verstanden werden. ${ }^{4}$ Er umschreibt den Prozess der Auflösung traditioneller sozialer Strukturen, der politischen Demokratisierung und gesellschaftlichen Partizipation. Demgegenüber wird Modernisierung mitunter lediglich auf einzelne Felder der sozialen Realität bezogen - meist verstanden als Prozess zunehmender Rationalisierung und Effizienzsteigerung. ${ }^{5}$ Problematisch ist es, wenn man von solchen Veränderungen in ge-

4 Vgl. Frei, Norbert: Wie modern war der Nationalsozialismus?, in: Geschichte und Gesellschaft 19, 1993, S. 367-387, hier S. 375-377.

5 Vgl. Schneider, Michael: „Volkspädagogik“ von rechts: Ernst Nolte, die Bemühungen um die „Historisierung“ des Nationalsozialismus und die „selbstbewusste Nation“, 
sellschaftlichen Teilsystemen unhinterfragt auf das Gesamtsystem schließt und diesem damit Modernität zuschreibt - der Teil also für das Ganze genommen wird.

Anknüpfend an diese Überlegungen zeigt die folgende Skizze beispielhaft, weshalb der Erste Weltkrieg nicht als modern charakterisiert werden sollte. Verwendet man den Begriff modern im Sinne von zum ersten Mal zum Einsatz kommend, dann stellt sich die Frage, welche Aspekte am Ersten Weltkrieg tatsächlich genuin neu waren bzw. warum sie in den zeitgenössischen Diskursen als solche angesehen wurden. Wie neuere Studien zeigen, verhinderte der verengte eurozentrische Blick der damaligen Eliten, dass die bereits vollzogenen Veränderungen in außereuropäischen Kriegen und den Kolonialkriegen von den europäischen Großmächten ausreichend wahrgenommen wurden. Nur deshalb erschienen die Entwicklungen auf dem europäischen Schlachtfeld in Ausmaß und Brutalität als überraschend neu. So nutzten die europäischen Armeen schon in den Kolonialkriegen das enorme Tötungspotenzial des Maschinengewehrs. Auch der Einsatz neuer Projektile, die aufgrund ihrer enorm gesteigerten Wirkung zunächst als zu barbarisch für ihre Verwendung in europäischen Konflikten angesehen wurden, lässt sich im Kontext der Kolonialkriege verorten. ${ }^{6}$ Der japanisch-russische Krieg von 1904/5 nahm mit seinem Zusammenspiel von Schützengraben, Stacheldraht, Artillerie und Maschinengewehr das Geschehen an der Westfront vollständig vorweg - besonders auch im Hinblick auf den Munitionsverbrauch und die Höhe der Verluste. ${ }^{7}$ Der industrialisierte Krieg erschien den Zeitgenossen besonders vor dem Hintergrund des wirkungsmächtigen Mythos vom heroischen Kampf Mann gegen Mann als etwas gänzlich Neues. Dabei waren die Mobilisierung von Massenheeren und der Einsatz von neuen Infanteriewaffen und Ferngeschützen bereits im 19. Jahrhundert Kriegsrealität. ${ }^{8}$ Fragt man nach den durch den Krieg hervorgerufenen Moderni-

[28.4.05], URL: http://www.fes.de/fulltext/historiker/00024toc.htm.

6 Vgl. Whiteclay Chambers II, John: The American Debate Over Modern War 1871-1914, in: Manfred F. Boemeke u.a. (Hg.): Anticipating Total War, Washington D.C. 1999, S. 241-279, hier S. 246f.; Trotha, Trutz von: „The Fellows Can Just Starve“. On Wars of „Pacification“ in the African Colonies of Imperial Germany and the Concept of „Total War", in: ebd., S. 415-435, hier S. 430-435.

7 Vgl. Whiteclay Chambers II (wie Anm. 6), S. 261f.

8 Die Waffentechnik erlebte bereits im Verlauf des 19. Jahrhunderts mehrere bedeutende Innovationsschübe, die deren Einsatzmöglichkeit und große Reichweite während des Ersten Weltkrieges maßgeblich vorbereiteten. Vgl. Trischler, Helmuth: Nationales Sicherheitssystem - nationales Innovationssystem. Militärische Forschung und Technik in Deutschland in der Epoche der Weltkriege, in: Bruno Thoß u. Hans-Erich Volkmann (Hg.): Erster Weltkrieg - Zweiter Weltkrieg : ein Vergleich, Paderborn 2002, S. 107-131, hier S. 113f;; Wilding, Peter: Krieg - Technik - Moderne: Die Eskalation der Gewalt im „IngenieurKrieg“. Zur Technisierung des Ersten Weltkrieges, in: Petra Ernst, Sabine A. Haring u. Werner Suppanz (Hg.): Aggression und Katharsis. Der Erste Weltkrieg im Diskurs der Moderne, Wien 2004, S. 163-186, hier S. 164. 
sierungsprozessen in Teilen der deutschen Gesellschaft, so zeigt sich die bis zum Kriegsende weitgehend ungebrochene Dominanz der alten Führungseliten. Die verantwortlichen Militärs passten ihre an vormodernen Idealen orientierten Handlungsmuster und überkommenen Standesvorstellungen nur widerstrebend an die Erfordernisse des industrialisierten Krieges an. ${ }^{9}$ So stießen beispielsweise die neuen Funktionseliten auf starke Vorbehalte im deutschen Offizierskorps. Techniker und Wissenschaftler wurden daher nur zögerlich in die strategische Planung und Entscheidungsfindung einbezogen. Zwei gut untersuchte Beispiele hierfür sind der Gaskrieg und der Krieg in der Luft. Es bedurfte der massiven Intervention durch den Wissenschaftler Fritz Haber, um die Oberste Heeresleitung (OHL) zum erstmaligen Einsatz von Giftgas im Stellungskrieg zu überreden. Und weiter versäumte es die OHL, ausreichende Truppenreserven für den ersten Chlorgaseinsatz bei Ypern bereitzustellen, um das tatsächlich erreichte Aufbrechen der erstarrten Frontlinie in einen strategischen Vorteil zu verwandeln. Die OHL hatte der neuen Waffe kein Vertrauen geschenkt. ${ }^{10}$ Auch der Einsatz von Flugzeugen im Ersten Weltkrieg verdeutlicht, dass die zweifellos innovative Entwicklung der Flugzeugtechnik durch die militärische Führung nicht aufgenommen wurde. Im Gegenteil begrenzten die deutschen Militärs durch ihre mangelnde Unterstützung den Spielraum der Flugzeugingenieure. ${ }^{11}$ Die Entstehung des Militärisch-Industriellen Komplexes und damit einhergehend Ansätze zu einer autarken Kriegswirtschaft, die durch die Kriegsrohstoffabteilung vorangetrieben wurden, sind ohne Zweifel Aspekte der Modernisierung der deutschen Wirtschaft. Das Beispiel der AEG zeigt jedoch, dass für die damalige Zeit hochmoderne Produktionsanlagen lediglich zur Herstellung eines vergleichsweise simplen Massenproduktes - nämlich der Granate - genutzt wurden. ${ }^{12}$ Auch die vermehrte Beschäftigung von Frauen in der Rüstungs-

9 Vgl. Epkenhans, Michael: Die Politik der militärischen Führung 1918: „Kontinuität der Illusionen und das Dilemma der Wahrheit", in: Jörg Duppler u. Gerhard P. Groß (Hg.): Kriegsende 1918. Ereignis, Wirkung, Nachwirkung, München 1999, S. 217-233, hier S. 219f.; Walle, Heinrich: Technikrezeption der militärischen Führung in Deutschland im 19. und 20. Jahrhundert, in: Michael Salewski u. Ilona Stölken-Fitschen (Hg.): Moderne Zeiten. Technik und Zeitgeist im 19. und 20. Jahrhundert, Stuttgart 1994, S. 93-118, hier S. $115 f$.

10 Vgl. Szöllösi-Janze, Margit: Fritz Haber 1868-1934. Eine Biographie, München 1998, S. 325.

11 Zur zögerlichen Nutzung der neu aufkommenden Luftfahrttechnik in der strategischen Planung der deutschen Militärs und dementsprechend geringen strategischen Wirkung s. Neitzel, Sönke: Zum strategischen Mißerfolg verdammt? Die deutschen Luftstreitkräfte in beiden Weltkriegen, in: Thoß/Volkmann (wie Anm. 8), S. 167-192, hier S. 168. Zum ambivalenten Verhältnis des deutschen Militärs gegenüber der modernen, industriellen Technik und dessen Ursachen siehe Wilding (wie Anm. 8), S. 170f.

12 Vgl. Deutsches Technikmuseum, Historisches Archiv, Firmenarchiv AEG-Telefunken, I.2.060 A Nr. 06072, Hauptversammlungen bis 1945, hier Bilanzpressekonferenz der AEG v. 9.2.1940 (anwesend H. Bücher und K. Boden), S. 10. 
industrie kann, wie neuere Untersuchungen belegen, nicht als Zeichen eines gesellschaftlichen Emanzipationsprozesses gewertet werden. Sie sollte vielmehr als ein bloßes Zugeständnis an den Arbeitskräftemangel gesehen werden. So bediente sich die Kriegswirtschaft der Instrumente und Methoden der industriellen Moderne, ohne selbst modern zu sein. ${ }^{13}$

Insgesamt bleibt festzuhalten, dass sich in Ansätzen neben den nichtmodernen, überkommenen Strukturen Elemente der Modernität entwickelten, wobei die retardierenden Elemente - zumindest in Deutschland - bis Kriegsende überwogen haben. Die Gleichzeitigkeit des Ungleichzeitigen scheint also das eigentliche Wesen des Ersten Weltkrieges auszumachen. Der Erste Weltkrieg kann somit nicht angemessen als modern charakterisiert werden. Er wirkte vielmehr als ein Katalysator gesellschaftlicher Modernisierungsprozesse, die sich erst ab November 1918 voll entfalteten. ${ }^{14}$

Die TV-Autoren vertreten dagegen die These, dass sich der Erste Weltkrieg grundlegend von allen bisherigen europäischen und weltweiten Kriegen unterscheide. Seine Modernität zeige sich besonders in den drei folgenden Aspekten: in der Einführung und Nutzung neuer, moderner Waffen, in der Entstehung des Militärisch-Industriellen Komplexes und in der Einbindung von Wissenschaftlern und Technikern in die Kriegsführung. In Anlehnung an die zeitgenössische Metaphorik vom Krieg als Maschine und den Soldaten als Arbeitern ${ }^{15}$ setzen sie die Modernität des Ersten Weltkrieges mit der Rationalisierung und Effizienzsteigerung der Kriegswirtschaft und Kriegsführung gleich. In der Erzählung nehmen Erfindung und Einsatz neuer Waffen einen sehr breiten Raum ein. Sowohl in der bildlichen Inszenierung wie auch im Kommentar wird immer wieder auf das wachsende Destruktionspotenzial und die damit einhergehende Brutalisierung des Kriegsgeschehens hingewiesen. Zusammenfassen lässt sich die Grundaussage der untersuchten Fernsehdokumentationen dahingehend, dass Technik und Wissenschaft als Antrieb der Kriegsmaschinerie eine neue Dimension entwickeln, in der sich der Erste Weltkrieg von allen vorangegangenen Kriegen unterscheide. ${ }^{16}$

Vergleicht man diese Thesen mit den eingangs angestellten Überlegungen, wird die verengte Perspektive der Filmemacher deutlich. In ihrer Dar-

13 Beispiele wären der Einsatz von Werkzeugmaschinen sowie Serienfertigung und vermehrte Frauenarbeit. Vgl. hierzu Reimann, Aribert: Der Erste Weltkrieg - Urkatastrophe oder Katalysator?, in: Aus Politik und Zeitgeschichte 29/30, 2004, S. 30-38, hier S. 38.

14 Vgl. Thoß, Bruno: Militärische Entscheidung und politisch-gesellschaftlicher Umbruch. Das Jahr 1918 in der neueren Weltkriegsforschung, in: Duppler/Groß (wie Anm. 9), S. 17-37, hier S. 18.

15 Kaufmann, Stefan: Kriegführung im Zeitalter technischer Systeme - Zur Maschinisierung militärischer Operationen, in: Militärgeschichtliche Zeitschrift 61, 2002, S. 337-367, hier S. 360f.

16 Die hier angesprochenen Thesen der TV-Autoren werden in der folgenden Filmanalyse exemplarisch entwickelt und durch Filmzitate belegt. 
stellung der scheinbar neuen Waffen und ihrer Auswirkungen auf das Kriegsgeschehen folgen sie weitgehend den oben kurz skizzierten zeitgenössischen Diskursen. Darüber hinaus transportieren gerade die bildlichen Darstellungen von Waffentechnik, Industrieanlagen und wissenschaftlichen Laboratorien den starken - hier nicht gebrochenen - Technikoptimismus und die Wissenschaftsgläubigkeit des wilhelminischen Kaiserreiches. ${ }^{17}$

In Bezug auf sozioökonomische Wandlungsprozesse weisen die Dokumentationen - explizit in den Kommentaren und implizit in den Bildern zwar auf einzelne Aspekte von Modernität hin. ${ }^{18}$ Sie thematisieren aber an keiner Stelle das dahinter liegende Spannungsverhältnis zwischen modernen und nicht-modernen Strukturen. Die als wesentlich erachtete Gleichzeitigkeit des Ungleichzeitigen wird also nicht problematisiert. Vielmehr fokussieren die TV-Autoren ihre ganze Darstellung auf die wissenschaftlichtechnische Entwicklung und nehmen damit letztlich den Teil für das Ganze.

\section{Analyse der filmischen Inszenierung von Wissenschaft und Technik}

Die Filmemacher nutzen die filmischen Darstellungen von Waffentechnik in zweierlei Hinsicht: ${ }^{19}$ Zum einen illustrieren sie ihre Geschichten über die Erfindung und Nutzung neuer Waffen mit zeitgenössischem Bildmaterial, das eben diese zeigt. Zum anderen verwenden sie dynamische Filmbilder von einzelnen Kriegsikonen - wie feuernde Artillerie und Granateneinschläge -, um die Gesamterzählung zu dramatisieren. ${ }^{20}$ Die Bilder sind in diesem Fall vollständig dekontextualisiert, stehen also in keinem erkennbaren Zusammenhang zum gerade erzählten Plot. Diese Bilder transportieren eine bis heute scheinbar ungebrochene Faszination von Waffentechnik und verleihen dem Krieg eine ästhetische Dimension. Anders als Flugzeuge, Giftgas oder Panzer thematisieren die Fernsehautoren Waffen der Artillerie nicht in einem eigenen, in sich geschlossenen Abschnitt. Vielmehr dienen entsprechende Filmsequenzen der durchgängigen Rhythmisierung und Strukturierung der Gesamterzählung: die Artillerie - so die Aussage von Bild und Kommentar trägt das Kriegsgeschehen und zugleich die Gesamterzählung.

17 Vgl. zur Gleichzeitigkeit von Fortschrittsoptimismus und kulturellem Skeptizismus im Deutschen Kaiserreich Orland, Barbara: Zivilisatorischer Fortschritt oder Kulturdeformation? Die Einstellung des Deutschen Kaiserreiches zur Technik (= Preprints zur Kulturgeschichte der Technik 1, 1998), [28.3.2005], URL: http://www.tg.ethz.ch/ dokumente/pdf_files/OrlandFORTSCHRITT.pdf, S. 4-7; Salewski, Michael: Technik als Vision der Zukunft um die Jahrhundertwende, in: Salewski/Stölken-Fitschen (wie Anm. 9), S. 77-91, hier besonders S. 79-81.

18 Ein Beispiel ist der Einsatz von Frauen in der Rüstungsindustrie: 1914-1918: Der Moderne Krieg, 01:03:21-01:03:44.

19 Die im Beitrag zitierten Filmbilder und -sequenzen sind repräsentativ für die gesamte Inszenierung der untersuchten Dokumentationen.

20 Hierzu zählt auch die Verwendung von Tonikonen wie z.B. Gewehrsalven und Granatendetonationen. 
Die zeitgenössische Filmpropaganda inszenierte Artilleriegeschütze als imposantes Kriegsgerät. ${ }^{21} \mathrm{Ob}$ die Kanone bedrohlich-einschüchternd oder offensiv auf den Betrachter wirkte, hing auch von der jeweils gewählten Kameraperspektive ab. Teilweise blickte der Betrachter gleichsam von vorne in den Kanonenlauf hinein - die Bedrohlichkeit der Waffe zeigte sich damit unmittelbar. In der Regel filmte die zeitgenössische Propaganda Kanonen aber entweder von der Seite oder schräg von hinten, so dass die Kamera den Lauf der Waffe entlang blickte bzw. der Flugbahn des Geschosses zu folgen schien. Die Bilder von Granatenexplosionen folgen einer wiederkehrenden Ästhetik: heraufspritzende Erde, aus der sich eine dunkle Rauchsäule erhob. Die große Zahl an Soldaten, die notwendig war, um schwere Artilleriegeschosse in Position zu bringen und zu bedienen, verstärkte den Eindruck der Wuchtigkeit des Kriegsgeräts. Auch blieben die Soldaten in der Mehrzahl der Aufnahmen gegenüber der riesigen Kanone eher klein und perspektivisch im Hintergrund..$^{22}$ Ein anderes, häufig wiederholtes Bildmotiv zeigt Soldaten, die in rascher Folge eine Kanone mit Munition laden und feuern. ${ }^{23}$ Die Propaganda versuchte, die Feuerkraft der eigenen Artillerie zu veranschaulichen.

Im Fall der Artillerieszenen ist die nachträgliche Rekombination des zeitgenössischen Filmmaterials besonders auffällig: Die Filmemacher verknüpfen in der Regel zwei bzw. drei Bildsequenzen miteinander. In der ersten Sequenz sieht man eine Kanone, die entweder gerade in Position gebracht, geladen oder abgefeuert wird. Oftmals werden mehrere Bilder unterschiedlicher Kanonen aneinander montiert, die hintereinander jeweils Granaten abfeuern - ein Sinnbild des nicht enden wollenden Trommelfeuers der Artillerie. In der nächsten Sequenz sieht man Granateinschläge; der Zusammenschnitt von Feuer und Einschlag generiert eine zusätzliche Dynamik, die dem ursprünglichen Filmmaterial fehlte. ${ }^{24}$

Die zahlreichen Filmszenen von Kanonen und Granatenexplosionen, welche die gesamte Erzählung durchziehen, stellen Kriegstechnik immer wieder in den Vordergrund. Die den Bildern innewohnende Dynamik und Ästhetik zieht die Aufmerksamkeit des Fernsehzuschauers auf sich.

21 Vgl. auch im Folgenden Paul, Gerhard: Bilder des Krieges - Krieg der Bilder: Die Visualisierung des Krieges in der Moderne, Paderborn 2004, S. 117-121 und Eisermann, Thilo: Pressephotographie und Informationskontrolle im Ersten Weltkrieg: Deutschland und Frankreich im Vergleich, Hamburg 2000, S. 107f. Vgl. zur Inszenierung von Waffen und Kriegsgerät als Zeichen der Überlegenheit Baumeister, Martin: „L'effet du réel“. Zum Verhältnis von Krieg und Film 1914 bis 1918, in: Bernhard Chiari, Matthias Rogg u. Wolfgang Schmidt (Hg.): Krieg und Militär im Film des 20. Jahrhunderts, München 2003, S. 245-268, hier S. 253f.

22 All diese Bildmotive finden sich exemplarisch in 1914-1918: Der Moderne Krieg, 00:02:0200:02:19.

23 Ebd., 00:41:11-00:41:19.

24 Exemplarisch ebd., 01:07:28-01:07:31. 
Die Aufnahmen der deutschen Kriegspropaganda stellten den Luftkrieg als heroische Schlacht dar, die mit der Realität der Materialschlachten wenig gemeinsam hatte. Der Blick auf das Schlachtfeld aus der Vogelperspektive sollte dem Betrachter die Distanz der neuen Waffengattung zum blutigen Kriegsgeschehen am Boden vor Augen führen. In der Luft - so suggerierten diese Bilder - habe der Krieg eine andere Qualität als am Boden. ${ }^{25}$ Aus dem Flugzeug gefilmte Kampfszenen zeigten die Besatzung, die gerade eine gegnerische Maschine mit einer gezielten Maschinengewehrsalve zum Absturz brachte. In dieser Darstellung bekam der Luftkrieg einen beinahe sportlichen Charakter; gegnerische und eigene Flugzeuge fochten einen Ringkampf aus. ${ }^{26}$

Dieses Bild wurde auch durch die Inszenierung der Piloten getragen. Die Propaganda stilisierte Manfred von Richthofen, aber auch andere damals berühmte Flieger dementsprechend zu Rittern der Lüfte. ${ }^{27}$ Sie filmte Richthofen bei der Vorbereitung seines nächsten Fluges - ein Soldat half ihm, seinen schweren, ledernen Fluganzug anzuziehen, er erklomm das Flugzeug, während weitere Personen ihm beim Anlegen des Sicherheitsgurtes assistierten. ${ }^{28}$ Darin klang das traditionelle Bildmotiv des Ritters an, der seine Rüstung anlegt und mit Hilfe seiner Knappen das Schlachtross besteigt.

Die Autoren der ARTE-Dokumentation übernehmen diese zeitgenössischen Diskurse weitgehend unverändert. Der Kommentar referiert die damalige Propagandasprache und verstärkt damit noch die Aussagen des verwendeten Bildmaterials; so perpetuieren sie den Mythos vom effizienten, sauberen Luftkrieg und der Besonderheit der Fliegergemeinschaft. Daneben eröffnen sie einen zweiten Diskurs, der weit über die zeitgenössische Darstellung hinausgeht. In Ermangelung aussagekräftigen Bildmaterials wird dieser fast ausschließlich durch den Kommentar getragen. Er weist auf die angeblich massenhafte Produktion von Flugzeugen durch eine rasch wachsende äußerst effizient fertigende Flugzeugindustrie hin. ${ }^{29}$ Das Spannungsverhältnis zwischen der sich vormodern gebenden Fliegertruppe und der durch den Krieg in ihrer Entwicklung gehemmten modernen Flugzeugindustrie sprechen sie nicht an. ${ }^{30}$

Die Entstehung des Militärisch-Industriellen Komplexes visualisieren die Filmemacher mithilfe von Filmmaterial aus der Rüstungsproduktion. Die in

25 Vgl. Paul (wie Anm. 21), S. 103f.

26 1914-1918: Der Moderne Krieg, 01:00:49-01:01:06 und 01:01:53-01:02:11.

27 Vgl. Hüppauf, Bernd: Kriegsfotografie, in: Wolfgang Michalka (Hg.): Der Erste Weltkrieg. Wirkung, Wahrnehmung, Analyse, München 1997, S. 875-905, hier S. 900.

28 1914-1918: Der Moderne Krieg, 01:01:13-01:01:40.

29 Ebd., 01:02:41-01:04:15.

30 Neitzel (wie Anm. 11), S. 169 u. Braun, Hans-Joachim: Krieg der Ingenieure? Technik und Luftkrieg 1914 bis 1945, in: Thoß/Volkmann (wie Anm. 8), S. 193-210, hier S. 195-197. Braun weist explizit darauf hin, dass für das Zurückfallen Deutschlands gegenüber der Entente im Flugmotorenbau ,die Produktion, nicht die Konstruktion und das technische Know-how, ... sich als Achillesferse [erwies].“ Ebd., S. 196. 
der Dokumentation gezeigten Darstellungen der unaufhörlich fortlaufenden Serienfertigung, der immensen Größe der Produktionsanlagen und Fertigungshallen wurden von der zeitgenössischen Filmpropaganda inszeniert, um die eigene industrielle Potenz und technische Überlegenheit zu verdeutlichen. Ein dazu eingesetztes Stilmittel waren Bildausschnitte, die so gewählt wurden, dass beispielsweise die Reihe der Granaten in der Fertigung aus dem Bild hinaus- und damit in die Unendlichkeit verwies. Die Nahaufnahme des Granatkarussells unter der Fräse deutete mit ihrer Kreisbewegung ebenfalls auf die niemals endende Munitionsproduktion hin. ${ }^{31}$ Die bei der Aufblende ins Bild rückende Frau wurde durch die Kameraperspektive und die Motivwahl zu einem menschlichen Anhängsel der Maschine. In einer in der Langfassung „Der moderne Krieg“ ergänzten Szene erscheinen die Frauen in der Granatproduktion der AEG in ihren repetitiven Bewegungen selbst vollständig maschinenhaft. ${ }^{32}$ Der Blickwinkel der Kamera in die Kanonenwerkstatt war wiederum so gewählt, dass die Halle möglichst lang und weit erschien; das großkalibrige Kanonenrohr im Bildvordergrund wirkt dadurch noch imposanter. ${ }^{33}$

Beide Filmsujets, die Faszination der Serienproduktion und die riesigen Dimensionen des Baus großer Anlagen bzw. Anlagenteile und die Art ihrer Inszenierung - Motivwahl, Bildausschnitt, Kamerastandpunkt -, standen ganz in der Tradition der frühen Industriefotografie, wie sie etwa von Krupp in Essen bekannt ist. ${ }^{34}$ Die Firma Krupp setzte als eine der ersten ihre Produktion von Kriegsgerät imposant fotografisch in Szene ${ }^{35}$ und stellte dessen zerstörerisches Potenzial dabei bewusst als Fortschritt heraus.

Die Aufnahmen der Munitionslager und -transporte wurden filmisch so in Szene gesetzt, dass sie dem damaligen Zuschauer die großen Vorräte der eigenen Granaten verschiedenen Kalibers und damit die eigene Überlegenheit buchstäblich vor Augen führten. Die Bildausschnitte geben den Blick nie auf die Ränder - die Begrenzung - der Stapel aufgeschichteter Granaten frei. ${ }^{36}$ Dies sollte die Zivilbevölkerung und die Soldaten als Betrachter der Bilder beruhigen, war doch der eigene Munitionsmangel eine ernsthafte und tödliche Bedrohung. Der als Abschluss der Sequenz gezeigte gigantische Berg verschossener Granathülsen symbolisiert darüber hinaus, mit welcher

31 Alptraum Verdun, 00:17:40-00:17:47.

32 1914-1918: Der Moderne Krieg, 00:21:30-00:21:38.

33 Alptraum Verdun, 00:18:00-00:18:05.

34 Vgl. die Fotografien der Granat- und Kanonenproduktion bei Krupp aus den Jahren 19021906 bei Lüdtke, Alf: Gesichter der Belegschaft. Porträts der Arbeit, in: Klaus Tenfelde (Hg.): Bilder von Krupp. Fotografie und Geschichte im Industriezeitalter, München 1994, S. 67-88, hier S. 81 u. 86f.; Wengenroth, Ulrich: Die Fotografie als Quelle der Arbeitsund Technikgeschichte, in: ebd., S. 89-104, hier S. 101.

35 Vgl. Tenfelde, Klaus: Geschichte und Fotografie bei Krupp, in: ders. (wie Anm. 34), S. 305-320, hier S. 308.

36 1914-1918: Der Moderne Krieg, 00:21:06-00:21:22. 
ungeheuren Feuerkraft man den Gegner eingedeckt hatte. ${ }^{37}$ Die von den heutigen Filmemachern eingesetzten Schnitte und Überblenden zwischen den Bildern aus der industriellen Fertigung, den Munitionsvorräten im Felde und den gebrauchten, leeren Granathülsen verbildlichen den Kommentar, der die Soldaten als Konsumenten und Opfer der Kriegsmaschinerie bezeichnet. ${ }^{38}$

Ingenieure und Techniker treten - im Gegensatz zu Wissenschaftlern in den Dokumentationen nur anonym in Erscheinung und werden bildlich durch ihre Erfindungen in Szene gesetzt. Die Erzählung über die Arbeit der Rüstungsingenieure erschöpft sich in einer Aufzählung von neu- bzw. weiterentwickelten Waffen: größere Artilleriegeschosse, Minen- und Flammenwerfer und Gas. Bildlich unterstützt wird diese Darstellung durch eine Aneinanderreihung von kurzen Filmszenen, in denen die jeweils im Kommentar erwähnten Waffen im Einsatz gezeigt werden. Die Standorte der Kameras sind oftmals ein deutliches Zeichen dafür, dass die Aufnahmen keine Gefechtssituationen, sondern Übungen oder sogar absichtlich inszenierte Waffenpräsentationen zeigen. Dazu passt auch eine Szene, in der französische Soldaten für die Kamera neben einer Granatenreihe posieren. Die Granaten wurden dazu wie Matroschkas ihrer Größe nach aufgereiht. Die Darstellung erinnert in ihrer Bildsprache an die fortschrittsgläubige Inszenierung von Produkten auf den Industrie- und Weltausstellungen der Vorkriegszeit. Die anwachsende Reihe bis hin zu mannshohen Geschossen sollte die Leistungsfähigkeit und Potenz der eigenen Rüstungstechnik symbolisieren. ${ }^{39}$ Der Kommentar reduziert die Tätigkeit der Ingenieure auf ein vermeintlich ökonomisches Gesetz, das lautet: „Maximales Blutbad zu minimalen Kosten““.

In all diesen Darstellungen dominieren wiederum Bilder technischer Artefakte. Die Tätigkeit der Ingenieure, ihre Rolle im Kriegsgeschehen und der daraus erwachsende Bedeutungszuwachs dieser neuen Funktionselite bleibt - ebenso wie die Konflikte mit den alten Führungseliten - ausgespart. ${ }^{41}$

Wissenschaft, im Kommentar als neue Antriebskraft der Kriegs- und Rüstungsmaschine bezeichnet, wird dagegen am Beispiel der Chemie aufwändig filmisch inszeniert. Dazu bedienen sich die Filmemacher stereotyper Bildikonen: beispielsweise der Blick in ein chemisches Laboratorium mit seinen Apparaturen, die Großaufnahme von Reagenzgläsern und Erlenmeyer-Kolben, in denen teilweise Flüssigkeiten brodeln und Gase aufsteigen. Dabei wechseln sich zeitgenössische Aufnahmen und heute nachgedrehte Szenen ab. ${ }^{42}$ Teilweise sind die Schnitte so gesetzt, dass dem Fernsehzuschauer das Gefühl vermittelt wird, in den nachgedrehten Szenen die Fortsetzung einer zeit-

37 Alptraum Verdun, 00:18:28-00:18:34.

38 1914-1918: Der Moderne Krieg , 00:49:25-00:50:50.

39 Sequenz in Alptraum Verdun, 00:30:15-00:31:41.

40 Ebd., 00:30:45-00:30:47.

41 Vgl. Braun (wie Anm. 30), S. 195 u. 209.

42 Gashölle Ypern, 00:17:29-00:18:04 u. 00:18:48-00:19:08. 
genössischen Aufnahme zu erleben. Mit einer einzigen Ausnahme lassen sich die gezeigten Bilder nicht der vom Kommentar beschriebenen Tätigkeit nämlich der Entwicklung von Giftgas - zuordnen. Vielmehr handelt es sich um typische Bildikonen der chemischen Wissenschaft und Industrie, die erst durch ihre Kontextualisierung dem Gaskrieg zugeordnet werden. Schließlich wird Wissenschaft durch den Chemiker Fritz Haber personifiziert. Die ausführliche Schilderung seiner Rolle im Gaskrieg - getragen von Aussagen vermeintlicher Zeitzeugen ${ }^{43}$ - und die dazu gezeigten Bilder seiner Person stilisieren ihn zum Sinnbild für den wissenschaftlich geführten Gaskrieg. Mangels entsprechender zeitgenössischer Filmaufnahmen behelfen sich die TV-Autoren hier mit dem Abfilmen von Fotografien. Dies ist ein Stilmittel, das in den Dokumentationen Tempo aus der Erzählung nehmen soll: häufig zur Darstellung von Toten auf dem Schlachtfeld. Um die Geschichte der Kampfgasentwicklung zu dynamisieren, schneiden die Filmemacher in eine Fotografie von Fritz Haber, die ihn mit einer Gruppe Militärs auf einem Feld zeigt, in den Bildvordergrund eine Filmaufnahme des Abblasens von Gas. ${ }^{44}$

Die Erzählung über den wissenschaftlich geführten Gaskrieg steht symbolisch dafür, dass erstmals Wissenschaft für den Krieg eine relevante Rolle spielt. Untermauert wird diese These durch die klischeehafte Aussage der Tochter von Fritz Haber: Ihr Vater sei der erste Wissenschaftler gewesen, der sich die Uniform angezogen habe. ${ }^{45}$

\section{Gründe für die mediale Konstruktion von Wissenschaft und Technik als Paradigma des Ersten Weltkrieges}

Die untersuchten Fernsehbeiträge orientieren sich in ihrer Machart an dem von Guido Knopp erfolgreich eingeführten neuen Stil für zeithistorische Dokumentationen. ${ }^{46}$ Dieser ist geprägt durch weitreichende Veränderungen in der Ästhetik und Dramaturgie der Inszenierung. Die klassische Rolle des Historikers tritt zurück, auch Fotografien und Zitate aus Dokumenten wer-

43 Neben tatsächlichen Zeitzeugen kommen auch deren Kinder zu Wort, die beispielsweise Tagebücher, Feldpostbriefe und Erzählungen ihrer Väter so paraphrasieren, dass der Zuschauer den Eindruck gewinnt, sie seien selbst dabei gewesen. Noch bedenklicher ist, dass die Filmemacher die Identität von Fachhistorikern und Biographen verschleiern und diese als vermeintliche Zeitzeugen präsentieren. So wird der Historiker Gerhard Kaiser als „Neffe eines deutschen Soldaten bei Ypern“ und der Haber-Biograph Dietrich Stoltzenberg als „Sohn eines Mitarbeiters von Fritz Haber“ vorgestellt. Vgl. zur neuen Rolle von Zeitzeugen in historischen Dokumentationen Buggeln, Marc u. Horn, Sabine: „Und Zeitgeschichte schien etwas zu sein, was in der Regel so aussah, daß Historiker vor Bücherregalen saßen“ - Gespräch mit Esther Schapira, in: Zeitschrift für Sozialgeschichte des 20. und 21. Jahrhunderts 17, 2002, S. 157-171, hier S. 166.

44 Sequenz in Gashölle Ypern, 00:21:12-00:21:22; vgl. zur Ästhetik der Bilder vom Gaseinsatz auch Hüppauf (wie Anm. 27), S. 895.

45 Gashölle Ypern, 00:22:06-00:22:25.

46 Vgl. Knopp, Guido u. Quandt, Siegfried (Hg.): Geschichte im Fernsehen. Ein Handbuch, Darmstadt 1988. 
den kaum eingesetzt. Stattdessen steigt der gezeigte Anteil von zeitgenössischen Filmaufnahmen stark an und persönliche Erlebnisse, von Zeitzeugen erzählt, treten in den Vordergrund. Damit soll dem Zuschauer ein besonderes $\mathrm{Ma}$ an Authentizität und Unmittelbarkeit vermittelt werden. ${ }^{47}$

Die bewegten Bilder kommen den Sehgewohnheiten der Fernsehzuschauer entgegen. Auch die Rhythmisierung der Erzählung durch schnelle Schnitte lehnt sich an die aktuelle Ästhetik des Fernsehens an. ${ }^{48}$ Die durchgehende Illustrierung der Erzählung mit zeitgenössischem Bildmaterial setzt dem Spielraum der heutigen Filmemacher aber enge Grenzen. Denn die Masse der Bilder transportiert - durch den Kommentar nur unzureichend gebrochen - die in ihnen eingravierten Diskurse der damaligen Filmproduzenten und ihrer Auftraggeber. Wie die zitierten Beispiele zeigen, ging es der Kriegspropaganda darum, die eigene technische Überlegenheit in Szene zu setzen. Die aufwändige und dramatische Inszenierung von Kriegsgerät bot sich hierfür besonders an.

Die TV-Autoren verzichten sowohl in ihrer Bildauswahl und -montage als auch in ihren Kommentaren auf eine quellenkritische Analyse des verwendeten Filmmaterials. So differenzieren sie beispielsweise nicht zwischen authentischen und gestellten Aufnahmen und thematisieren auch nicht die problematische Verwendung letzterer. Sie folgen vielmehr mit ihrer - von den Bildern präfigurierten - These von der herausragenden Rolle von Wissenschaft und Technik dem filmischen Blick der Kriegspropaganda. Die zeitgenössische Technikfaszination bleibt ungebrochen.

Die untersuchten Dokumentationen sind auch unter einem erinnerungsgeschichtlichen Blickwinkel interessant. ${ }^{49} \mathrm{Im}$ Gegensatz zur britischen und französischen Erinnerungskultur ist der Erste Weltkrieg im kollektiven Gedächtnis der Deutschen seiner Eigenständigkeit heute weitgehend entklei-

47 Buggeln/Horn (wie Anm. 43), S. 162f. und Bösch, Frank: Das „Dritte Reich“ ferngesehen. Geschichtsvermittlung in der historischen Dokumentation, in: Geschichte in Wissenschaft und Unterricht 50, 1999, S. 204-220, hier S. 218.

48 Vgl. Linne, Karsten: Hitler als Quotenbringer - Guido Knopps mediale Erfolge, in: Zeitschrift für Sozialgeschichte des 20. und 21. Jahrhunderts 17, 2002, S. 90-101, hier S. 97; Klöss, Erhard: Die Last der Bilder - Geschichte im Fernsehen, in: Klaus Füßmann, Heinrich Theodor Grüttner u. Jörn Rüsen (Hg.): Historische Faszination. Geschichtskultur heute, Köln, Weimar, Wien 1994, S. 189-193, hier S. 191.

49 Siegfried Kracauer hat zuerst auf die Rolle von Filmen bei der Konstruktion von Mentalität und Geschichtsbildern einer Gesellschaft hingewiesen. Vgl. Kracauer, Siegfried: Von Caligari zu Hitler. Eine psychologische Geschichte des deutschen Films, Frankfurt a.M. ${ }^{4} 1999$, S. 11-13. Nach Günter Riederer ermöglicht die Analyse von Filmen und historischen Dokumentationen Aussagen über gesellschaftlich gültige Normen, Haltungen und Werte der Zeit; vgl. Riederer, Günter: Was heißt und zu welchem Ende studiert man Filmgeschichte? Einleitende Überlegungen zu einer historischen Methodik der Filmanalyse, in: Chiari/Rogg/Schmidt (wie Anm. 21), S. 85-106, hier S. 94. Dazu auch Tschirbs, Rudolf: Film als Gedächtnisort, in: Geschichte in Wissenschaft und Unterricht 54, 2003, S. 578 594, hier S. 579. 
det. So wurde er erst jüngst von Hans-Ulrich Wehler zur bloßen Generalprobe des Zweiten Weltkrieges degradiert. ${ }^{50}$ Die Filmemacher schließen sich in ihrer Erzählung dieser ahistorischen Deutung ebenfalls an; so endet die Schilderung des Luftkrieges mit dem Verweis darauf, dass diese neue Waffe ihr ganzes Potenzial erst im Zweiten Weltkrieg entfalten konnte. Und die Filmbilder des Jagdfliegers Hermann Göring werden mit seiner zukünftigen Rolle als Hitlers Paladin kommentiert. ${ }^{51}$ Ältere Diskurse, wie zum Beispiel die Fischer-Kontroverse, sind nicht mehr präsent. Die Erinnerung an den Ersten Weltkrieg scheint also durchaus offen zu sein für neue Bedeutungszuschreibungen, von denen der Erste Moderne Krieg nur eine mögliche ist.

Und schließlich greift die in den TV-Dokumentationen häufig verwendete Maschinenmetaphorik aktuelle erinnerungspolitische Diskurse auf. In Teilen der deutschen Medien dominieren seit einigen Jahren Opfernarrative in der Darstellung des Zweiten Weltkrieges. ${ }^{52}$ Diese Perspektive wird offenbar auch auf den Ersten Weltkrieg übertragen. ${ }^{53}$ Die Logik einer autonomen Kriegsmaschinerie folgt scheinbar nur ihrer eigenen Gesetzmäßigkeit und entbindet damit den Einzelnen von Verantwortung und Schuld. Letztlich werden alle gleichermaßen zu Opfern des Krieges. Symptomatisch dafür ist zum einen die Ausblendung des Kriegsausbruchs und seiner Ursachen in beiden Dokumentationen..$^{54}$ Zum anderen werden in der ARD-Folge „Trau-

50 Vgl. Wehler, Hans-Ulrich: Der zweite Dreißigjährige Krieg. Der Erste Weltkrieg als Auftakt und Vorbild für den Zweiten Weltkrieg, in: Spiegel special 1, 2004, S. 138-143. Wissenschaftler und Publizisten waren von jeher darum bemüht, den Ersten Weltkrieg epochal zu verorten. Gerade in der bundesdeutschen Historiographie wird der Erste Weltkrieg häufig als bloßes Vorspiel für den Zweiten Weltkrieg gedeutet, dem kein eigener, historisch bedeutsamer Platz zukommt. Vgl. zu den britischen, französischen und amerikanischen Deutungen des Ersten Weltkrieges Reimann (wie Anm. 13), S. 38. Zur Konstruktion des Mythos Erster Weltkrieg in Großbritannien vgl. Korte, Barbara: Mediale Konstruktionen des ,Mythos“ Erster Weltkrieg: Eine Betrachtung zum kulturellen Gedächtnis Britanniens Ende des zwanzigsten Jahrhunderts, in: Annette Simonis u. Linda Simonis (Hg.): Mythen in Kunst und Literatur: Tradition und kulturelle Repräsentation, Köln 2004, S. 125-139.

51 1914-1918: Der Moderne Krieg, 01:02:17-01:02:37.Vgl. zur Überlagerung der Erinnerung an den Ersten Weltkrieg durch den Zweiten Weltkrieg Hirschfeld, Gerhard: Kriegserlebnis, Mentalität und Erinnerung. Der Erste Weltkrieg in der deutschen und internationalen Geschichtsschreibung, in: Ernst/Haring/Suppanz (wie Anm. 8), S. 367-386, hier S. 367.

52 Vgl. Ther, Philipp: Soll und Haben. Warum das deutsche Kaiserreich kein Nationalstaat war, in: Le Monde diplomatique, 13.5.2005, S. 16-17; vgl. auch Assmann, Aleida: Erinnerung als Erregung. Wendepunkte der deutschen Erinnerungsgeschichte, in: Wolf Lepenies (Hg.): Wissenschaftskolleg - Institute for Advanced Study - zu Berlin. Jahrbuch 1998/ 99, Berlin 2000, S. 200-220, hier S. 215.

53 Vgl. dazu auch Rother, Rainer: Die Erfahrung des Ersten Weltkrieges und der deutsche Film, in: Thoß/Volkmann (wie Anm. 8), S. 821-838, hier S. 838.

54 Die beiden Filmemacher Heinrich Billstein und Mathias Haentjes reduzieren den Kriegsausbruch auf den lapidaren Kommentar: „Seit Wochen hat sich die Krise in Europa zugespitzt. Jetzt entlädt sich die Spannung.“ 1914-1918: Der moderne Krieg, 00:05:58-00:06:20. 
ma Versailles“ die Deutschen - durch die nicht weiter kommentierten Aussagen von Zeitzeugen - zu wehrlosen Opfern der annexionistischen Kriegszielpolitik der Entente-Mächte stilisiert.

\section{Fazit}

Folgt man diesen beiden Deutungsangeboten, führt der selbst auferlegte Zwang, in erster Linie mit historischem Bildmaterial Geschichte zu erzählen, sowie die fehlende Quellenkritik zu der verengten Perspektive der Filmemacher auf den Ersten Weltkrieg. Die prominente Position der Technik in den zeitgenössischen Kriegsbildern kommt zudem heutigen - medial konstruierten - Vorstellungen vom technisierten Krieg entgegen..$^{55}$ Und schließlich knüpfen die TV-Autoren an derzeitige erinnerungsgeschichtliche Diskurse an. Die Eingrenzung des modernen Krieges auf Wissenschaft und Technik führt - wie dargelegt - zur verkürzten Darstellung des Ersten Weltkrieges. So ist zum einen die Darstellung des technisierten Schlachtfeldes, auf dem Menschen als bloße Anhängsel der Kriegsmaschinen arbeiten, eine Verharmlosung komplexer Technisierungsprozesse. Zum anderen marginalisiert die Ausblendung sozioökonomischer Prozesse die Handlungsspielräume und damit auch Verantwortlichkeiten der beteiligten Akteure. Im populären Geschichtsbild der untersuchten zeithistorischen Dokumentationen bleibt die Gleichzeitigkeit des Ungleichzeitigen hingegen ausgeblendet.

Anschrift der Verfasser: Silke Fengler, RWTH Aachen, Lehrstuhl für Geschichte der Technik, Kopernikusstraße 16, D-52074 Aachen, Email: fengler@histech.rwth-aachen.de; Stefan Krebs, RWTH Aachen, Lehrstuhl für Geschichte der Technik, Kopernikusstraße 16, D-52074 Aachen, Email: krebs@histech.rwth-aachen.de.

Die Kriegsgründe bleiben dagegen vollständig ausgeklammert. Susanne Stenner widmet in ihrem Beitrag „Mythos Tannenberg“, dem ersten Teil der ARD-Serie, der Darstellung der Kriegsgründe lediglich 40 Sekunden. Sie verweist auf das deutsche Flottenbauprogramm und die Aufrüstungspolitik der europäischen Großmächte. Mythos Tannenberg, 00:02:20-00:03:00.

55 In einer besonders prägnanten Sequenz werden der Abschuss eines Eisenbahngeschützes und der senkrechte Blick aus der Luft auf eine gewaltige Explosion ineinander geblendet. 1914 - 1918: Der moderne Krieg, 01:00:06-01:00:12. Diese Szene lehnt sich an die Bildästhetik der Berichterstattung über den ersten Irakkrieg an. Dort wurden die Aufnahmen so montiert, dass der Abschuss von seegestützten Marschflugkörpern und die Zielbildvideos amerikanischer Kampfflugzeuge aus ihren Handlungszusammenhängen gelöst und miteinander verbunden wurden. Vgl. Paul (wie Anm. 21), S. 399. 
Wissenschaftliche Leitung:

Gerhard Dohrn-van Rossum

Friedrich Klemm (1965-1975)

Wolfgang König (1987-2003)

Karl-Heinz Ludwig (1976-1997)

Conrad Matschoß (1909-1941)

Kurt Mauel (1974-1994)
Reinhold Reith

Wilhelm Treue (1965-1992)

Ulrich Troitzsch (1976-2000)

Adam Wandruszka (1965-1969)

Ulrich Wengenroth

Karin Zachmann

\section{Redaktion: Dr. Astrid Schürmann, Katharina Zeitz M.A.}

Die Zeitschrift TECHNIKGESCHICHTE schließt nach einem Registerband 31 (1965) mit dem Band 32 an das Jahrbuch Technikgeschichte, Beiträge zur Geschichte der Technik und Industrie, Bd. 1 bis 30 (1909 bis 1941), an.

TECHNIKGESCHICHTE veröffentlicht Beiträge über die geschichtliche Entwicklung der Technik in ihren wissenschaftlichen, gesellschaftlichen, wirtschaftlichen und politischen Zusammenhängen. Die Aufsätze dieser Zeitschrift werden begutachtet (refereed journal).

\section{Anschrift der Redaktion:}

Katharina Zeitz M.A., Technische Uni-

versität Berlin, TEL 12-1

Ernst-Reuter-Platz 7, D-10587 Berlin

Tel. 030 / 31424085

Fax $030 / 31425962$

E-Mail Technikgeschichte@TU-Berlin.de

Anschrift des Verlags:

edition sigma e.Kfm.

Karl-Marx-Str. 17, D-12043 Berlin

Tel. 030 / 6232363

Fax 030 / 6239393

E-Mail verlag@edition-sigma.de

Hinweise für Autor/inn/en:

Beachten Sie bitte die Hinweise für die Manuskripteinreichung in diesem Heft. Die Zusendung von Büchern zur Rezension an die Redaktionsanschrift ist willkommen, Rezensionsexemplare können jedoch nicht zurückgesandt werden.

Anzeigenverwaltung: edition sigma (Anschrift siehe oben). Zurzeit gilt Anzeigentarif 1/2005

Druck: Rosch-Buch GmbH, Scheßlitz Gedruckt auf umweltfreundlichem, alterungsbeständigem Papier.

Printed in Germany.

ISSN 0040-117X

TECHNIKGESCHICHTE im Internet:

www.edition-sigma.de/TG
Bezugsbedingungen:

Jahresabonnement (4 Hefte): 78,00 Euro; für Studierende*: 46,80 Euro. - Einzelheft: 21,50 Euro. - Vorzugspreis* für Mitglieder des Vereins Deutscher Ingenieure, der Deutschen Gesellschaft für Geschichte der Medizin, Naturwissenschaft und Technik und der GeorgAgricola-Gesellschaft: Jahresabo 70,20 Euro, Einzelheft 19,50 Euro. - Sonderpreise* für Mitglieder der Gesellschaft für Technikgeschichte: siehe www.edition-sigma.de/TG (* Studien- bzw. Mitgliedschaftsnachweis erforderlich.)

Alle Preisangaben: zuzügl. Versandkosten ab Verlagsort; einschl. gesetzl. Mehrwertsteuer.

Abonnements verlängern sich um jeweils ein Jahr, es sei denn, sie werden spätestens sechs Wochen vor Ende des Bezugszeitraums schriftlich beim Verlag gekündigt. - Die zur Verwaltung von Abonnements erforderlichen Datenwerden beim Verlag unter Beachtung der Vorschriften des Bundesdatenschutzgesetzes elektronisch gespeichert.

Copyright: edition sigma e.Kfm.

Alle in dieser Zeitschrift veröffentlichten Beiträge sind urheberrechtlich geschützt. Kein Teil dieser Zeitschrift darf außerhalb der engen Grenzen des Urheberrechtsgesetzes ohne vorherige schriftliche Zustimmung des Verlags reproduziert, übersetzt oder verbreitet werden. 